\title{
Papel da radiologia intervencionista no atendimento ao paciente politraumatizado
}

\author{
The role of interventional radiology in trauma patient care
}

\author{
Francisco Cesar Carnevale ${ }^{1}$, Airton Mota Moreira ${ }^{2}$
}

\begin{abstract}
Carnevale FC, Moreira AM. Papel da radiologia intervencionista no atendimento ao paciente politraumatizado / The role of interventional radiology in trauma patient care. Rev Med.(São Paulo). 2011 out.-dez:;90(4):201-14.
\end{abstract}

\begin{abstract}
RESUMO: O trauma é causa importante de morte e afastamento do trabalho. A Radiologia Intervencionista por meio de técnicas minimamente invasivas pode ser útil para politraumatizados estáveis com lesões vasculares ou de órgãos sólidos. Apesar de exigir treinamento, máquinas e materiais adequados, pode proporcionar melhores resultados. Lesões intra-abdominais devem ser suspeitadas no trauma pélvico e/ou torácico com instabilidade. Hematomas, equimoses ou petéquias devem ser correlacionadas ao trajeto vascular e órgãos. O lavado peritoneal, tomografia, ressonância magnética e FAST (focused assesment with sonography for trauma) podem ser utilizados. Angiografias não são ideais para traumas abdominais agudos por não excluirem lesões associadas, mas, no território periférico são úteis para planejamento. Apesar da necessidade cirúrgica para casos graves, condutas conservadoras podem determinar melhor evolução em muitos casos. Nas lesões vasculares abdominais e orgãos maciços, angioplastia com ou sem stent pode ser utilizada nas lesões intimais e transmurais, e embolização nos sangramentos ativos. Lesões hepáticas podem ser conduzidas conservadoramente, por meio da embolização percutânea ou cirurgicamente. A embolização esplênica e renal deve ser considerada para pacientes selecionados. $\mathrm{Na}$ região cervical, são alternativa para lesões carotídeas de difícil acesso, acima do ângulo da mandíbula e petrosas. Por sua vez, stents revestidos ou endopróteses expandiram as indicações terapêuticas na aorta abdominal e torácica. Pequenas lesões das extremidades, assintomáticas e não oclusivas, podem ser tratadas conservadoramente. Lesões de membros e pélvicas necessitam estabilização óssea para controle. A indicação para embolização baseia-se na condição hemodinâmica e presença de sangramento. Maiores evidências comparando estas técnicas à cirurgia, ainda são necessárias.
\end{abstract}

DESCRITORES: Trauma; Radiologia intervencionista; Politraumatizado; Lesão vascular; Angiografia; Embolização; Stent

\begin{abstract}
Trauma is a leading cause of death worldwide and a leading cause of disability. Trauma is a serious public health problem with significant social and economic costs. While advances in trauma care and trauma care systems have improved patient outcomes, research shows that Interventional Radiology (IR) can advance trauma care for stable, multiple trauma patients including those with vascular and solid organs lesions. Although requiring training, special machines and materials, IR may provide better results. Intra-abdominal injury should be suspected in instable pelvic / thoracic trauma cases. Bruising, petechiae or ecchymosis should be correlated to the vascular topography and organs. The peritoneal lavage, computed tomography, magnetic resonance and FAST (Focused Assesment with Sonography) may be necessary. Angiogram should not be indicated for acute abdominal trauma diagnosis because it can't exclude associated lesions, but in the peripheral vascular area may be useful for planning. Despite surgery for severe cases, non-surgical treatment may determine a better outcome in many cases. The indication for embolization is based on the hemodynamic status and presence of active bleeding. Vascular and intra abdominal organ lesions may be treated by stenting, especially in cases of intimal and transmural vascular lesions or by embolization in bleeding cases. Liver injury can be conducted conservatively, surgically or by percutaneous embolization. The splenic and renal embolization should also be considered for selected patients. Small vascular extremity lesions, asymptomatic and nonocclusive, can be treated conservatively. In the neck region, IR is a good alternative to carotid lesions with difficult surgical access, above the angle of the mandible or petrous region. In turn, the use of covered and bare stents in the thoracic and abdominal aorta expands the treatment options. Pelvic and limb injuries require bone stabilization for adequate control. Further evidence comparing these techniques to surgery, are still needed.
\end{abstract}

KEYWORDS: Trauma; Interventional radiology; Polytrauma; Vascular injury; Angiography; Embolization; Stent

1. Médico, Doutor em Ciências, Chefe do Serviço de Radiologia Intervencionista do Hospital das Clínicas da Faculdade de Medicina da Universidade de São Paulo - HCFMUSP.

2. Médico, Doutor em Ciências, Assistente do Serviço de Radiologia Intervencionista do Hospital das Clínicas da Faculdade de Medicina da Universidade de São Paulo - HCFMUSP.

Endereço para correspondência: Francisco C. Carnevale. InRad-HCFMUSP. Av. Dr. Enéas de Carvalho Aguiar, 255 - Cerqueira César, São Paulo, SP, CEP: 05403-001. E-mail: fcarnevale@uol.com.br. 


\section{N T RO D U ÇÃ O A O T R A T A M E N T O ENDOVASCULAR DAS LESÕES TRAUMÁTICAS}

Was últimas décadas observou-se trauma, sobretudo relacionados aos acidentes automobilísticos e armas de fogo. O trauma tornou-se causa principal de morte entre jovens e é responsável pelo alto índice de afastamento do trabalho, superando câncer e doenças cardiovasculares.

Procedimentos "minimamente invasivos" em Radiologia Intervencionista vêm sendo utilizados de forma isolada ou associada para reduzir complicações de cirurgias abertas. Uma gama de procedimentos está disponível para o tratamento de lesões vasculares e não-vasculares ocorridas na comunidade e em estradas, ou ainda iatrogênicas após biópsias ou decorrentes de outros procedimentos médicos ${ }^{1}$.

Em grandes centros de trauma nos Estados Unidos, o médico intervencionista está integrado ao corpo clinico. Nestes pronto-socorros são tratadas lesões pélvicas, torácicas, vasculares, viscerais e cervicais numa média de 3 a 5 procedimentos por semana. A aplicabilidade das técnicas intervencionistas exige a disponibilidade de 24 horas e treinamento específico em Radiologia Intervencionista por parte do médico, assim como máquinas de fluoroscopia e materiais para o tratamento. Um importante fator para o sucesso é o serviço de resgate que, aliado a uma boa rede de acesso, permite a chegada em tempo hábil do paciente. Outro aspecto refere-se à organização dos centros e integração entre equipes, como clínicos, cirurgiões, radiologistas diagnósticos e intervencionistas para a escolha da melhor forma de tratamento.

No Brasil, o trauma é causa importante de óbito nas primeiras quatro décadas de vida, representando grande desafio social, econômico e produzindo forte impacto na mortalidade. Segundo dados governamentais e da Sociedade Brasileira de Atendimento Integrado ao Traumatizado (SBAIT), em 2004 ocorreram cerca de 150 mil mortes por trauma e estimativas indicam cerca de 800.000 internações anuais (apenas nos hospitais públicos). Na cidade de São Paulo, o serviço de resgate e o SAMU responderam a 30.000 chamados decorrentes de algum evento relacionado. Os custos econômicos envolvidos superam nove bilhões anualmente.

A utilização da Intervenção no trauma, inicialmente proposta para embolização do sangramento retroperitoneal associado a fraturas pélvicas, descrita por Landmark et al. ${ }^{2}$ do Massachusetts General Hospital nos Estados
Unidos, ganhou fama rapidamente. Nos dias atuais, a abordagem endovascular no trauma inclui o tratamento de órgãos maciços (hepáticos, esplênicos ou renais), pélvicos e dos membros, assim como de outros locais menos comuns, por meio de drenagens, embolizações e técnicas de restauro do fluxo vascular com "stents" revestidos e não revestidos. A divulgação destas técnicas pelos Radiologistas Intervencionistas, principalmente em nível emergencial, é de suma importância por oferecer forma opcional de tratamento com ótimos resultados ${ }^{2}$.

\section{MECANISMOS DO TRAUMA}

A identificação correta do mecanismo de lesão é essencial para a escolha mais apropriada da opção diagnóstica e terapêutica.

A. Trauma vascular periférico

Lesões vasculares em ambiente urbano se devem comumente a ferimentos penetrantes (facas ou projéteis). Acidentes automobilísticos e quedas são causas mais comuns das lesões contusas. Estas últimas envolvem maior morbidade, sobretudo se associadas a fraturas, luxações ou esmagamentos de tecidos moles ou nervoso ${ }^{3}$.

B. Trauma abdominal vascular visceral

O trauma visceral responde por 13 a $15 \%$ dos casos de acidentes fatais e contribui para altas taxas de mortalidade tardia por sepse. Decorrem de diversos mecanismos (Tabela 1) e são divididos em grupos:

a) traumatismos penetrantes

b) traumatismos fechados ou contusos.

O reconhecimento destes grupos é de extrema importância, para a escolha da conduta diagnóstica e terapêutica assim como avaliação do prognóstico.

TABELA 1. Etiologia das lesões abdominais

\begin{tabular}{ll}
\hline \multicolumn{1}{c}{ Penetrante } & \multicolumn{1}{c}{ Fechada } \\
\hline & $\begin{array}{l}\text { Acidentes automobilísticos } \\
\text { de altura }\end{array}$ \\
Projétil de arma de fogo & Explosões \\
Ferimento por arma & Atropelamentos \\
branca & Agressões \\
& Esmagamentos \\
\hline
\end{tabular}

Lesões penetrantes significativas ocorrem em $80 \%$ dos ferimentos por projétil de arma de fogo. $\mathrm{Na}$ Tabela 2 estão descritas as lesões mais frequentes após o traumatismo abdominal penetrante. 
TABELA 2. Traumatismo abdominal penetrante: lesões mais frequentes

\begin{tabular}{|ll|}
\hline Órgão & $\%$ \\
\hline Fígado & 37 \\
Intestino delgado & 26 \\
Estômago & 19 \\
Cólon & 17 \\
Lesão vascular & 13 \\
Lesão de retroperitônio & 10 \\
\hline
\end{tabular}

A grande maioria dos ferimentos penetrantes, principalmente provocados por projéteis de arma de fogo, deve ser tratada cirurgicamente em função das altas taxas de lesões intraperitoneais.

A incidência dos traumatismos abdominais fechados tem aumentado consideravelmente em grandes centros urbanos, onde o acidente automobilístico é o mais comum ${ }^{4}$ (Figura 1 ). Órgãos parenquimatosos são acometidos frequentemente devido à súbita transferência de energia após o impacto, determinando lacerações e rupturas (Figura 2). As lesões abdominais mais frequentes estão descritas na Tabela 3.

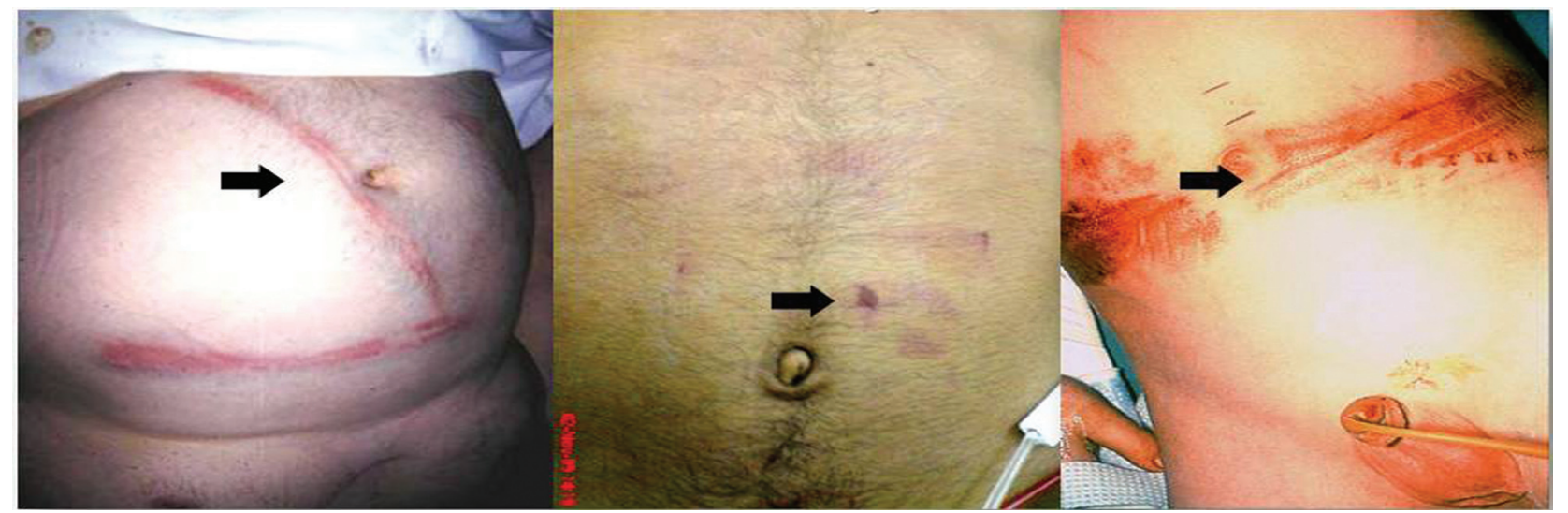

Figura 1. Escoriações e equimoses da parede abdominal em vítimas de acidente automobilístico urbano que podem sugerir trauma de órgãos intra-abdominais. Nos detalhes (setas) observam-se sinais de súbita transferência de energia (marcas de cinto de segurança e contusões)

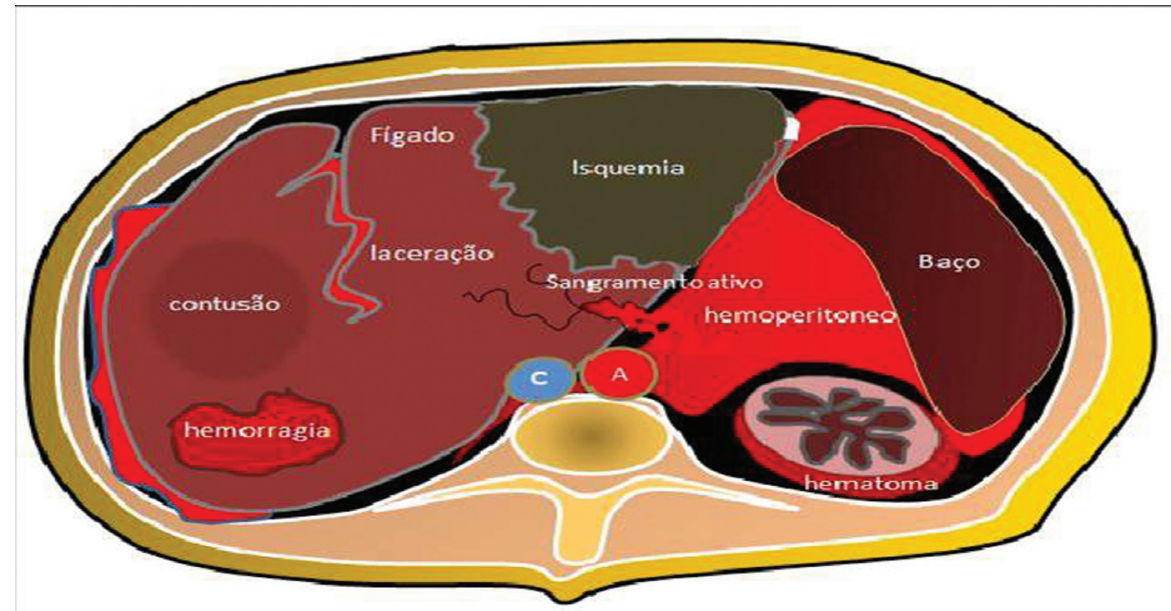

Figura 2. Representação esquemática de alguns dos tipos de lesões de órgãos internos observadas no trauma abdominal fechado

Tabela 3. Lesões mais frequentes no traumatismo abdominal fechado

\begin{tabular}{lc}
\hline Órgão & $\%$ \\
\hline Baço & 42 \\
Fígado & 35 \\
Diafragma & 05 \\
Intestinos & 05 \\
Rim & 03 \\
\hline
\end{tabular}

\section{ATENDIMENTO INICIAL E EXAME CLÍNICO DO PACIENTE POLITRAUMATIZADO}

Pacientes politraumatizados devem ser minuciosamente examinados quanto à existência de fatores de risco imediatos por meio do algoritmo A-B-C-D do ATLS para determinar permeabilidade das vias aéreas, perfusão e correção de déficits neurológicos. Alterações são corrigidas conforme a ordem de importância. 
A ressuscitação cardiorrespiratória é iniciada por meio de proteção à via aérea, ventilação, acesso venoso central e correção da volemia. Abordagens secundárias e exames físicos mais completos serão postergados. A avaliação dos sinais vitais, administração de fluidos e analgesia são particularmente importantes para casos de fraturas ou traumas crânio espinhais dolorosos.

A entubação orotraqueal é necessária nos casos de angústia respiratória grave, dispnéia ou sinais de choque.

A inspeção deve buscar hematomas, equimoses ou petéquias e correlacioná-las ao trajeto de vasos ou topografia de órgãos intra-abdominais onde o trauma pode determinar hemorragias graves.

A palpação, ausculta e percussão podem ser úteis na localização de pontos de dor, fluidos, gases ou massas. A dilatação de vasos superficiais da parede abdominal pode indicar o aumento da pressão intra-abdominal.

Exames físicos isolados do abdome, principalmente nos traumas contusos, são subjetivos e podem apresentar resultados ambíguos. Estudos retrospectivos holandeses demonstraram alta taxa de exames falso-negativos em $45 \%$ dos pacientes politraumatizados atendidos no setor de emergência, número que se eleva para $84 \%$ naqueles com fraturas múltiplas. Por este motivo, recomenda-se que pacientes com histórico de trauma abdominal devam ser avaliados sistematicamente por meio de métodos de imagem a fim de descartar lesões intraabdominais clinicamente significativas ${ }^{2}$.

A investigação diagnóstica abdominal inicial, em casos suspeitos, consiste do lavado peritoneal diagnóstico (LPD) ou tomografia computadorizada (TC) realizados com diferentes protocolos e contraste intravenoso, oral e retal. Entretanto, o exame denominado FAST, tradução da sigla inglesa para "focused assesment with sonography for trauma" pode ser realizado pelo próprio médico do setor de emergência em tempo médio de dois minutos. Este tipo de avaliação pode ser considerada extensão do exame clínico por ser objetiva, direcionada à detecção da presença ou ausência de hemoperitôneo, devendo ser considerada no diagnóstico inicial.

Pacientes hemodinamicamente instáveis que apresentaram reversão do quadro após medidas iniciais de ressuscitação, aqueles sob risco de apresentarem lesões vasculares ou abdominais graves e aqueles com exames não sugestivos de sangramento ativo evoluindo estáveis devem ser avaliados por meio do exame vascular periférico e abdominal, exames laboratoriais e exames de imagem seriados não invasivos ou invasivos para decisão terapêutica².

\section{Sinais clínicos de lesão arterial no trauma vascular periférico:}

a) Concretos: ausência de pulso, palidez, frialdade, dor, parestesia, poiquilotermia, sangramento ativo, hematoma pulsátil ou em expansão, frêmito ou sopro audível;

b) Duvidosos: história de sangramento ativo no local do trauma, proximidade da ferida penetrante ou contusa com a artéria principal, hematoma não pulsátil ou déficit neurológico.

Sinais concretos tornam necessária abordagem urgente. $O$ estudo angiográfico deve ser realizado preferencialmente quando ainda houver viabilidade do membro e na ausência de sangramentos ativos. As arteriografias intra-operatórias podem ajudar no planejamento cirúrgico.

A natureza insidiosa das lesões vasculares traumáticas de extremidade torna necessária investigação adicional uma vez que o exame clínico normal não exclui sua presença ${ }^{3}$.

Apesar da cirurgia ser um tratamento clássico para lesões vasculares traumáticas, a conduta conservadora é possível para lesões arteriais pequenas assintomáticas e não oclusivas ou identificadas acidentalmente por métodos de imagem. Apesar de alguns autores sugerirem seu tratamento imediato, abordagens conservadoras não-operatórias utilizando critérios clínicos e radiológicos de seguimento seria possível, uma vez que a resolução ou estabilização destas lesões pode ocorrer em cerca de 90\% dos casos $^{3}$.

Estes critérios incluem: velocidade;

a) Lesão produzida por projétil de baixa

b) Critério de imagem: Mínima ruptura arterial $(<5 \mathrm{~mm})$ irregularidades da íntima e pseudoneurismas não evolutivos;

c) Critério de imagem: Circulação distal intacta;

d) Ausência de sangramento ativo;

e) Sinais clínicos de trauma vascular visceral.

As lesões traumáticas de órgãos intraabdominais podem apresentar evolução insidiosa, situação em que a adequada avaliação clínica, hemodinâmica, laboratorial e por meio de exames de imagem seriados pode ser fator decisivo para a indicação terapêutica ${ }^{4}$.

As lesões viscerais subclínicas devem ser suspeitadas em casos de associação com traumas pélvicos e/ou torácicos graves, principalmente se há instabilidade hemodinâmica com resposta transitória aos fluidos.

Segundo Peitzman et al. ${ }^{4}$, em pacientes hemodinamicamente estáveis com lesões contusas 
hepáticas, esplênicas ou renais, o tratamento conservador imediatamente após o trauma, pode determinar uma melhor evolução clinica.

Na presença de sinais de extravasamento do contraste, pseudoaneurismas em lesões hepáticas, renais ou esplênicas de alto grau, hemoperitôneo, hematomas em progressão nas vísceras maciças ou hematomas pélvicos retroperitoneais em expansão nas tomografias seriadas de controle, o tratamento deve ser iniciado rapidamente. As lesões viscerais de alto grau podem ser tratadas de forma menos invasiva por meio de técnicas endovasculares percutâneas ${ }^{4}$.

\section{ESTUDO ANGIOGRÁFICO NO TRAUMA}

\section{A. Indicações e contra-indicações}

$\mathrm{Na}$ indicação de estudos angiográficos, deve-se levar em consideração o risco e o tempo necessários para sua realização, assim como as condições clínicas e hemodinâmicas do paciente. Os exames de imagem têm maior valor na detecção e tratamento de lesões ocultas e nas localizações de difícil acesso cirúrgico.

As arteriografias estarão contra-indicadas em casos onde há necessidade de exploração cirúrgica imediata por conta de outras lesões com maior prioridade ${ }^{5}$. Contra-indicações relativas para arteriografia incluem história de alergia a contrastes iodados, asma ou disfunção renal.

\section{B. Achados angiográficos no trauma}

1. Trauma vascular periférico: Sinais angiográficos fortemente sugestivos de lesão vascular devem ser considerados na decisão da terapêutica ${ }^{6}$ (Figura 3).

a) Extravasamento de contraste (seja para o exterior, cavidades ou contido, como os pseudoaneurismas);

b) Estreitamento da coluna de contraste (lesão intimal, mural ou extrínseca);

c) Dilatação vascular (ruptura intimal com preservação da media e adventícia ou nas fistulas artério-venosas crônicas);

d) Defeitos de enchimento ("flap" intimal, hematoma mural, êmbolo, trombo ou corpo estranho);

e) Estagnação do meio de contraste;

f) Fistulas artério-venosas agudas;

g) Oclusão vascular (trombose).
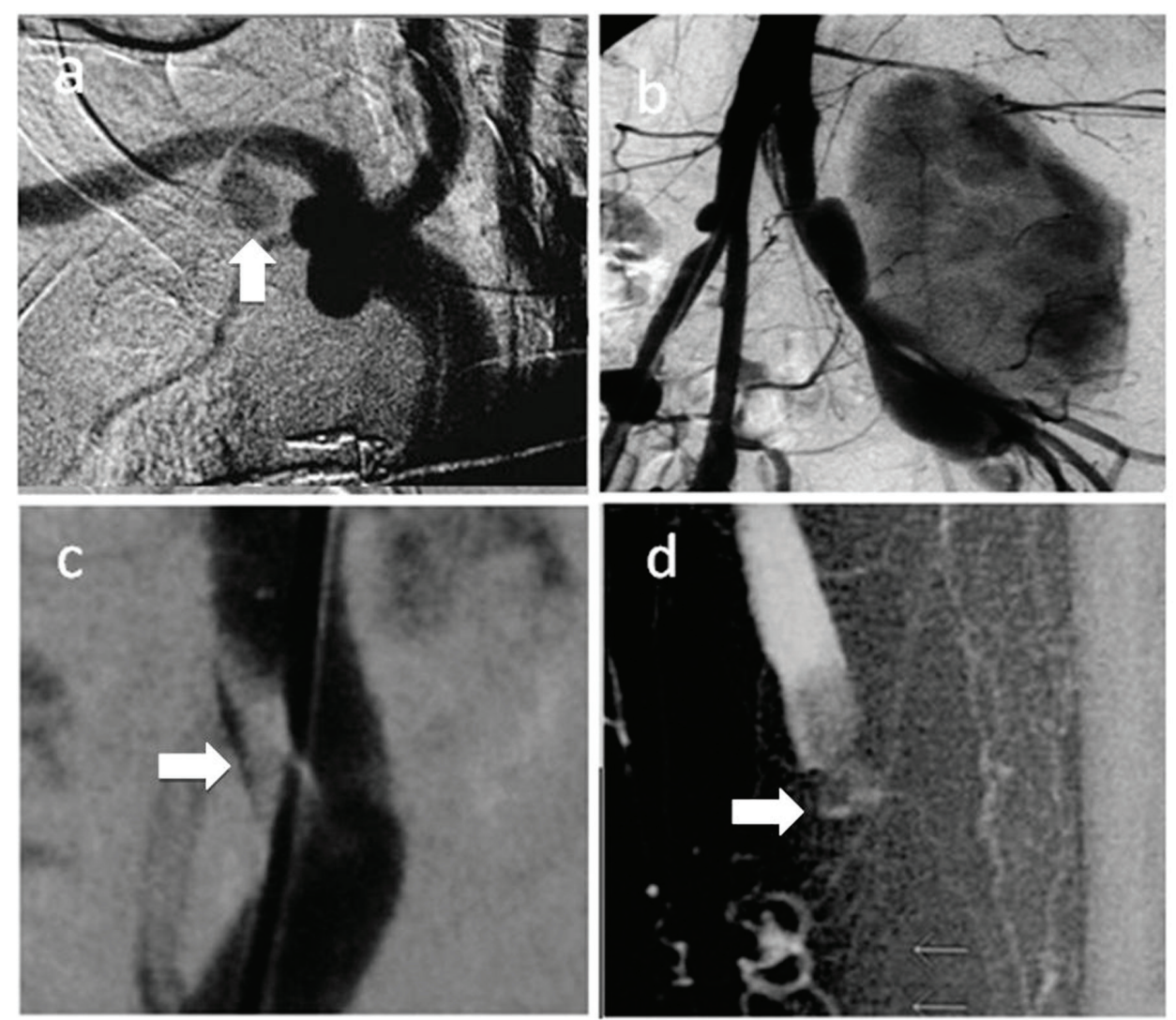

Figura 3. Sinais angiograficos sugestivos de lesão vascular. Em (a) observa-se extravasamento de contraste em lesão arterial da subclávia direita (seta), (b) pseudoaneurisma arterial ilíaco direito (seta), (c) defeito de enchimento intra-luminal sugestivo de "flap" intimal (seta), hematoma mural, êmbolo, trombo ou corpo estranho e em (d) oclusão vascular com estagnação (seta) do meio de contraste (trombose). 
Carnevale, Moreira. Papel da radiologia intervencionista no atendimento ao paciente.

\section{Trauma vascular visceral}

Apesar de ser padrão "ouro" para o diagnóstico de lesões vasculares, a angiografia não é o exame ideal para avaliação inicial de casos de trauma abdominal agudo pois, não permite excluir outros tipos de lesões associadas como as de vísceras ocas $^{7}$. Outros exames como a ultrassonografia (US), tomografia computadorizada (TC) e ressonância magnética $(\mathrm{RM})$ apresentam melhores taxas de sensibilidade.

Nas lesões de vísceras maciças, além dos achados angiográficos clássicos relacionados às lesões vasculares, outros comoáreas parenquimatosas hipovasculares e desvio ou deformidade no contorno visceral normal, podem sugerir lesão.

\section{PROCEDIMENTOS BÁSICOS E MATERIAIS EM RADIOLOGIA INTERVENCIONISTA PARA O TRA- TAMENTO DO TRAUMA}

Os principais tipos de intervenções endovasculares aplicáveis aos casos de trauma incluem basicamente a embolização e angioplastia com o uso de "stents" revestidos ${ }^{8}$.

O termo "embolização" significa oclusão vascular mediante introdução intraluminal de corpos
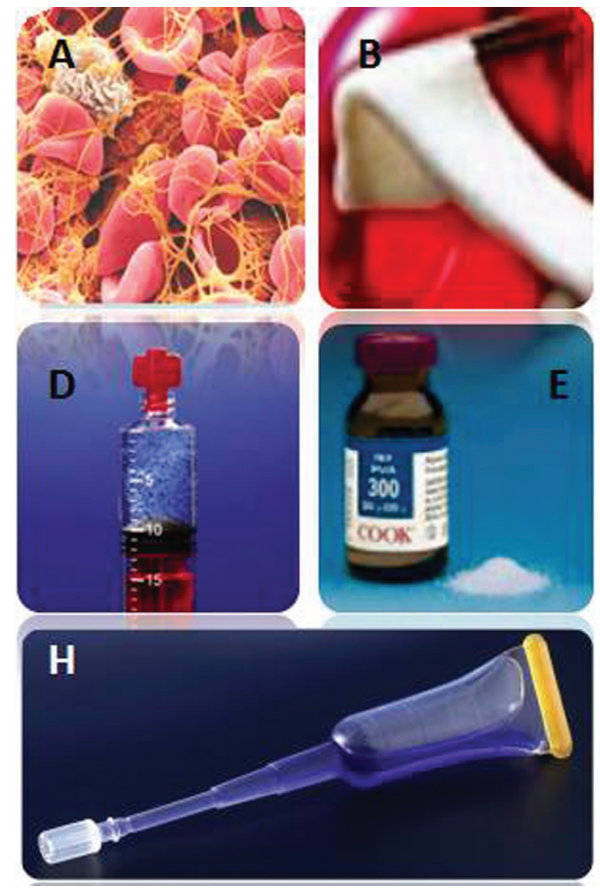

Figura 4. Agentes embolizantes. Em (a) coágulo autólogo; (b) Espuma de gelfoam; (c) Alcool etílico; (d) Microesferas acrílicas; (e) Partículas de polivinilalcool; (f) Molas metálicas fibradas; (g) Balões destacáveis, (h) Cianoacrilato; (i) Onix e (j) Plugs metálicos

A embolização permite a ação sobre focos de sangramento ativo evitando a necessidade de transfusões, da realização de cirurgias concomitantes estranhos ou tecidos com o intuito de interromper o fluxo sanguíneo mecanicamente, induzir trombose e produzir reação inflamatória (Figura 4). As artérias envolvidas no sangramento devem ser identificadas e podem ser ocluídas por meio do uso de diferentes agentes com variadas características próprias. De uma forma geral, estes agentes podem apresentar ação embolizante temporária ou permanente, serem particulados, líquidos, sólidos ou metálicos e apresentarem outras características embolizantes (Tabela 4).

Tabela 4. Classificação genérica e exemplos de agentes embolizantes

\begin{tabular}{ll}
\hline Tipo de agente & exemplo \\
\hline Biológico & $\begin{array}{l}\text { Coágulo autólogo e } \\
\text { tecidos } \\
\text { Gelfoam }\end{array}$ \\
Hemostático absorvível & $\begin{array}{l}\text { PVA, esferas, molas, } \\
\text { balão destacável } \\
\text { Cianoacrilato e Onyx } \\
\text { Polímero fluido }\end{array}$ \\
Esclerosante tecidual & $\begin{array}{l}\text { Alcool, ethamolin e } \\
\text { glicose } \\
\text { Alcool, ethamolin e } \\
\text { glicose }\end{array}$ \\
\hline
\end{tabular}
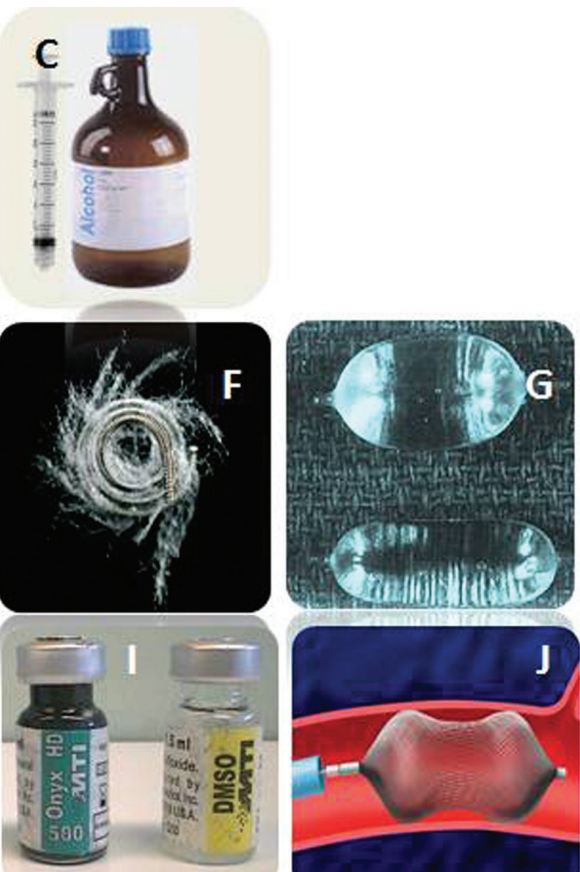

emergenciais e permite abordagens cirúrgicas em caráter eletivo. No caso dos pseudoaneurismas, a embolização previne a rotura e permite salvar o órgão acometido. 
Alguns princípios básicos de embolização devem ser respeitados com o fim de reduzir os riscos e obter o máximo de sucesso técnico, como:

1.Obter cateterismo seguro e estável;

2.Embolizar a favor do fluxo;

3. Utilizar técnica coaxial para pequenos vasos;

4.Não trabalhar com aparelhos ou imagens de má qualidade;

5.Embolizar somente o necessário.

Mesmo observando cuidados técnicos, o risco de embolia paradoxal pode determinar limites para o uso desta técnica na urgência. Faz-se necessário avaliar adequadamente a relação entre o vaso sangrante e a área de suprimento vascular ou o órgão associado. Na duvida de qual agente utilizar, pode-se utilizar agentes temporários, uma vez que o uso de agentes metálicos (molas) apesar de eficaz, apresenta caráter definitivo e irreversível.

Atualmente, novos agentes embolizantes vêm sendo desenvolvidos agregando melhores características, como maior regularidade no tamanho, compressibilidade e expansibilidade, maior trombogenicidade, possibilidade de liberação controlada ou escolha do tempo de polimerização adequado. Esta evolução, aliada à melhoria das técnicas de embolização, trouxe excelentes resultados para o uso deste recurso terapêutico (Figura 4).

Os "stents" revestidos foram originalmente desenvolvidos para o tratamento da doença arterial aneurismática. $\mathrm{Na}$ área do trauma podem ser utilizados como opção para o fechamento de vazamentos arteriais ou venosos, refazendo o conduto vascular e mantendo a perfusão do leito distal. No caso dos revestidos, além da menor flexibilidade, o processo de liberação costuma ser um pouco mais complexo que o do seu equivalente não revestido. Seu uso está bem aceito para vasos como a aorta, nos casos de roturas e dissecções, mas sua utilização torna-se difícil nas situações emergenciais envolvendo vasos de pequeno calibre e áreas de muita tortuosidade ${ }^{8}$.

Em algumas situações, o trauma pode determinar lesão da parede arterial seguida de delaminação, trombose e isquemia distal. Nestes casos, pode-se transpor a área rôta com fio-guia, seguindo-se o implante de stent com célula aberta para o restabelecimento do fluxo.

\section{TRATAMENTO ESPECIFICO DAS LESÕES VASCULARES E VISCERAIS}

\section{A. Lesões vasculares cervicais}

Embolizações, angioplastias com "stents" revestidos e oclusão vascular temporária têm sido liberalmente utilizadas em lesões de difícil acesso cirúrgico como lesões da carótida interna acima do segundo corpo vertebral cervical, acima do ângulo da mandíbula ou da sua porção petrosa.

As lesões da artéria carótida externa ou de seus ramos (facial, lingual, maxilar, occipital ou auricular) podem cursar com sangramentos importantes. Nestes casos, a embolização superseletiva pode ser indicada com sucesso, uma vez que a ligadura cirúrgica pode ser ineficaz, por conta da rica rede colateral $^{9}$.

As lesões intimais da artéria carótida interna, resultado da hiperextensão cervical, fraturas da base do crânio ou traumas penetrantes apresentam alta morbidade, por conta do alto risco de embolização ou trombose. Os "stents" têm sido utilizados nestes casos, mas seu uso liberal ainda carece de estudos randomizados de seguimento em longo prazo (Figura 5).

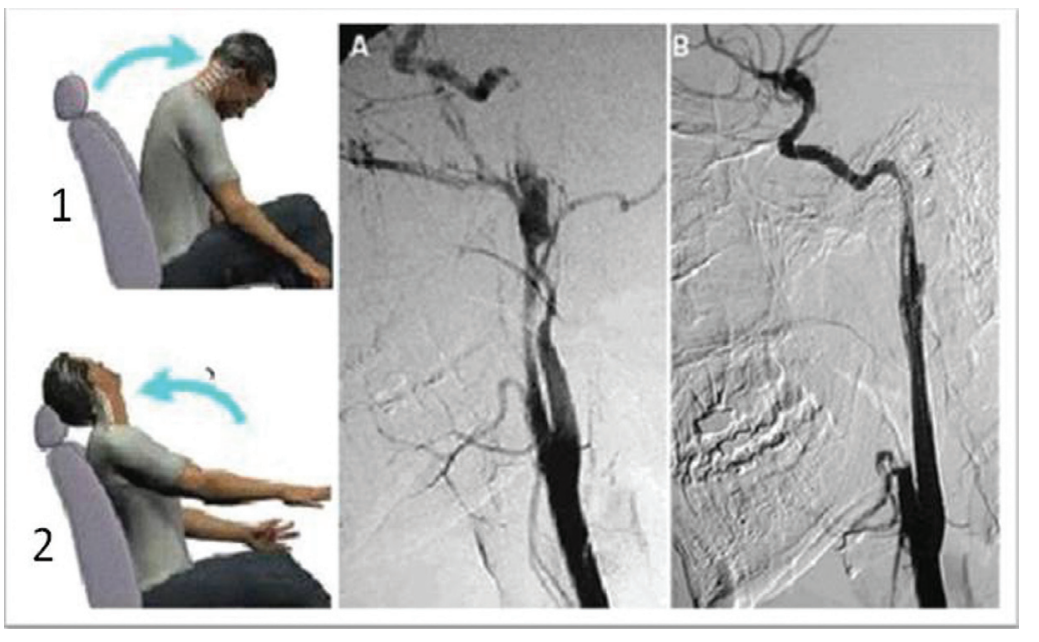

Figura 5. Imagem esquemática do efeito das forças de aceleração (1) e desaceleração (2) na região cervical durante acidente automobilístico. Em (A) angiografia carotídea esquerda onde se observa lesão da artéria carótida interna com trombose e em (B) sua correção por meio do implante de dois "stents" metálicos. Fonte: wwww.hemodinam adt.com.ar/journals/journal. 
Em casos de sangramentos ativos da artéria vertebral, a embolização é o tratamento de escolha. A necessidade de ocluir uma das vertebrais pode não se constituir em problema maior, na medida em que houver boa irrigação a partir da artéria contralateral. Nestes casos, a mola é o agente embolizante de escolha. Os segmentos proximal e distal à lesão devem ser embolizados, pois embolizações proximais podem ser insuficientes para conter o sangramento. Para lesões não oclusivas e não sangrantes o papel da embolização ainda não está bem estabelecido. Testes de oclusão ajudam a definir conduta ${ }^{9}$.

\section{B. Lesões vasculares torácicas}

As lesões traumáticas da aorta torácica são as principais causas de morte em politraumatizados nos Estados Unidos. Apesar dos avanços cirúrgicos e cuidados intensivos pós-operatórios, a mortalidade permanece inalterada se comparada aos dados da década de 50, com mortalidade de $85 \%$ no local do acidente e cerca de $75 \%$ nas primeiras duas semanas após o trauma. A ruptura clássica ocorre no istmo aórtico, um centímetro abaixo da origem da artéria subclávia esquerda, além de outros locais como a aorta ascendente, aorta descendente e abdominal, que são menos acometidos ${ }^{5}$.

O uso de "stents" revestidos ou endopróteses tem expandido o papel da radiologia intervencionista. Seu uso expandiu-se para o tratamento de oclusões arteriais crônicas, aneurismas periféricos e lesões vasculares traumáticas, sobretudo da aorta torácica e seus ramos. No caso da aorta torácica, anatomia distorcida pelo hematoma, presença de falso aneurisma ou hipertensão venosa em decorrência de fistulas arteriovenosas são fatores complicadores para o tratamento cirúrgico. A técnica endovascular permite abordagem à distância e reduz a morbimortalidade sobretudo, para pacientes críticos ${ }^{5}$ (Figura 6).

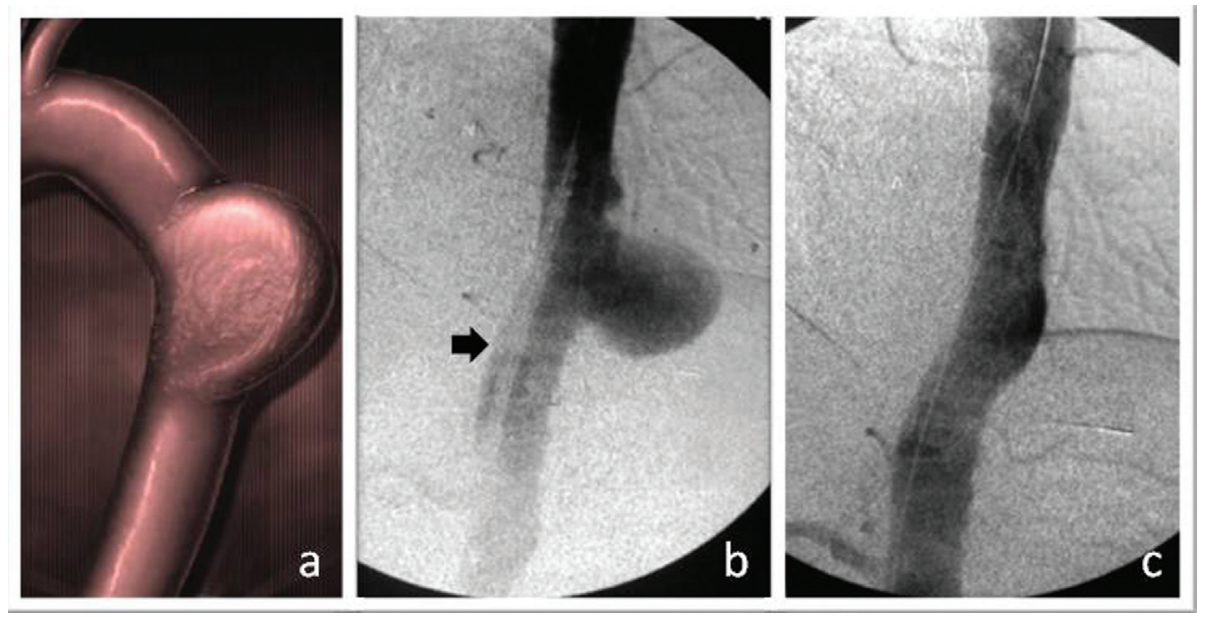

Figura 6. (a) Representação esquemática da dilatação aneurismática focal da parede da aorta torácica como em casos pós-traumáticos; (b e c) Detalhe da aortografia durante o tratamento da lesão similar (seta), sequela de trauma vacular, por meio do implante de endoprótese revestida.

\section{Lesões vasculares abominais}

As opções terapêuticas endovasculares estão limitadas aos casos de lesão da aorta abdominal e/ou das artérias ilíacas. Estes pacientes são, em geral, vitimas de ferimentos penetrantes nos quais a condição hemodinâmica pode abreviar a indicação cirúrgica e reduzir a indicação de métodos de imagem.

Apesar das lesões da aorta abdominal raramente passarem despercebidas, sua identificação tardia pode ser indicação para o tratamento endovascular, assim como para lesões traumáticas iatrogênicas como dissecções, oclusões, perfurações e rupturas arteriais ${ }^{9}$.

Lesões arteriais lombares podem estar associadas a fraturas pélvicas e serem identificadas durante estudos angiográficos aórticos. Nestes casos, pode-se lançar mão da embolização, com especial atenção para o risco de lesões arteriais medulares associadas.

No trauma abdominal fechado, lesões por desaceleração podem ser responsáveis por lesões da artéria renal, tronco celíaco, artéria hepática, esplênica e até mesmo de ramos mesentéricos. Dentre estes, a lesão da artéria renal é a mais comum 4 .

O tipo de lesão observada pode variar de rupturas, dissecções a oclusão. O encontro de infartos viscerais ou hematomas em TC de pacientes com relato de trauma abdominal fechado sugere a sua presença.

O tratamento endovascular pode incluir 
angioplastias com ou sem stent no caso de lesões intimais, angioplastia com "stents" revestidos nas lesões transmurais com sangramento ativo, recanalização das oclusões arteriais agudas ou a embolização de sangramentos intraparenquimatosos ativos (Figura 7).

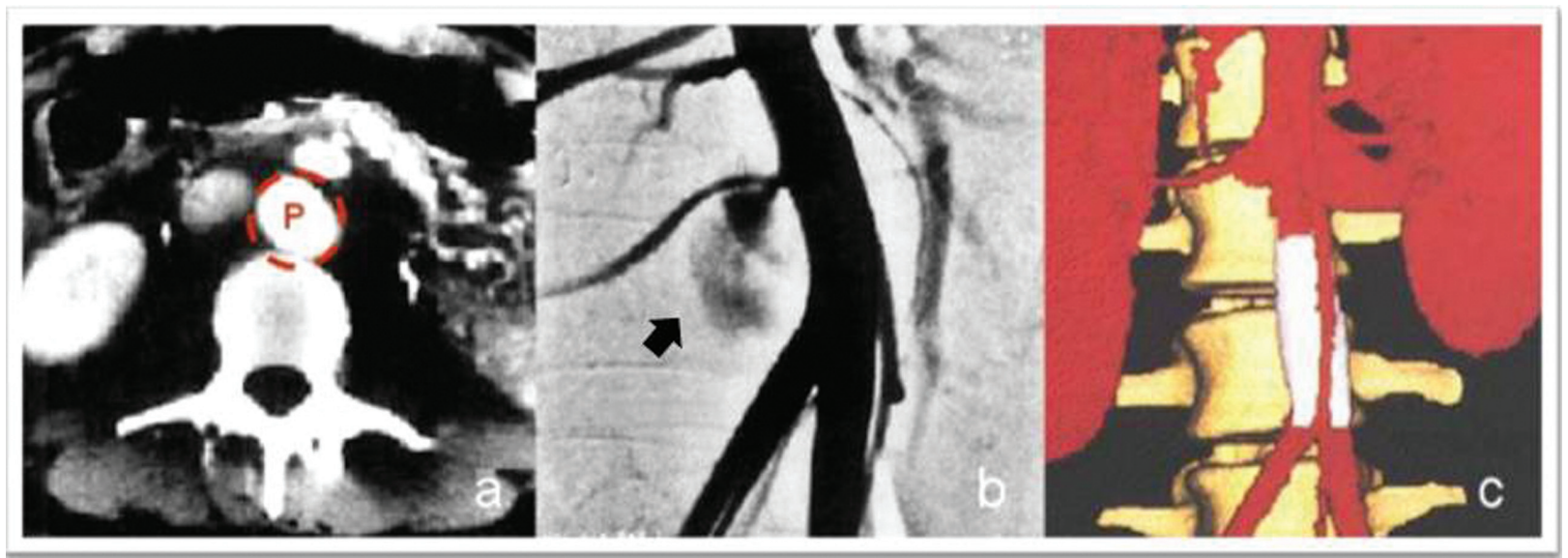

Figura 7: Em (a) Corte axial tomográfico (detalhe) onde se observa pseudoaneurisma (p vermelho) retroaórtico. Em (b) aortografia com extravasamento de contraste (seta) e em (c) reconstrução tomográfica da aorta abdominal em 3D demonstrando correção endovascular com endoprótese reta revestida

Fonte: Scharrer Pet al. Emergent endoluminal repair of delayed abdominal aortic rupture after blunt trauma. Endovasc Surg 1998, S 134-137.

\section{Lesões vasculares de extremidades}

a. Extremidades superiores: Lesões arteriais braquiais são mais frequentes dentre lesões dos membros superiores e seu diagnóstico raramente necessita de angiografia. Os espasmos arteriais podem simular lesões, principalmente na presença de fraturas ou contusões. Nestes casos, a angiografia pode diferenciar o espasmo da oclusão. Neste território, técnicas endovasculares como embolização possuem papel limitado, devido aos bons resultados e fácil acesso da abordagem cirúrgica. A abordagem de lesões do antebraço difere daquela da artéria braquial. A presença de três vasos permite a embolização de um deles em diversas circunstâncias, sem risco para a viabilidade do membro ${ }^{9}$.

b. Extremidades inferiores: Técnicas endovasculares percutâneas, como embolização, utilizadas em casos de sangramentos musculares, e oclusão temporária com balão, podem ser empregadas como métodos pré-operatórios.

Os pseudoaneurismas iatrogênicos da artéria femoral podem ser tratados tanto por compressão local como por angioplastia com "stents" revestidos. Sempre que possível, deve-se preservar a artéria femoral profunda que é via colateral importante na doença obstrutiva crônica do território íliofemoral e fêmoro-popliteo. Sua embolização também pode ser realizada em casos de fístulas artériovenosas crônicas ou na presença de feridas inguinais contaminadas. Nestes casos, molas podem ser posicionadas proximal e distalmente à lesão. De extrema importância também é a preservação da artéria femoral comum, pelo fato de ser uma região com possibilidade de realização de diferentes enxertos ou anastomoses vasculares. Assim, deve-se evitar o implante de "stents" ou endopróteses nesta topografia ${ }^{10}$.

A arteriografia é imprescindível para o planejamento cirúrgico, principalmente no território genicular e distal (Figura 8). O uso de técnicas endovasculares nestas lesões encontra-se bem estabelecido, podendo-se optar pelos acessos contralateral ou ipsilateral e pelo cateterismo seletivo anterógrado ou retrógrado. No caso do tratamento dos ramos geniculares, surais e artérias distais sangrantes, a cateterização superseletiva seguida de embolização é possível e necessária em muitos casos. Podem ser utilizados agentes embolizantes como molas ou gelfoam.

\section{E. Traumatismos hepáticos}

A mortalidade nos traumas hepáticos é de aproximadamente $10 \%$, podendo alcançar $25 \%$ no caso da associação com outras lesões. Os critérios de seleção sugeridos são apresentados na Tabela 5. As lesões hepáticas podem ser classificadas conforme o grau de lesão parenquimatosa, biliar ou vascular, a partir de onde se pode planejar adequadamente a conduta terapêutica ${ }^{11}$. 


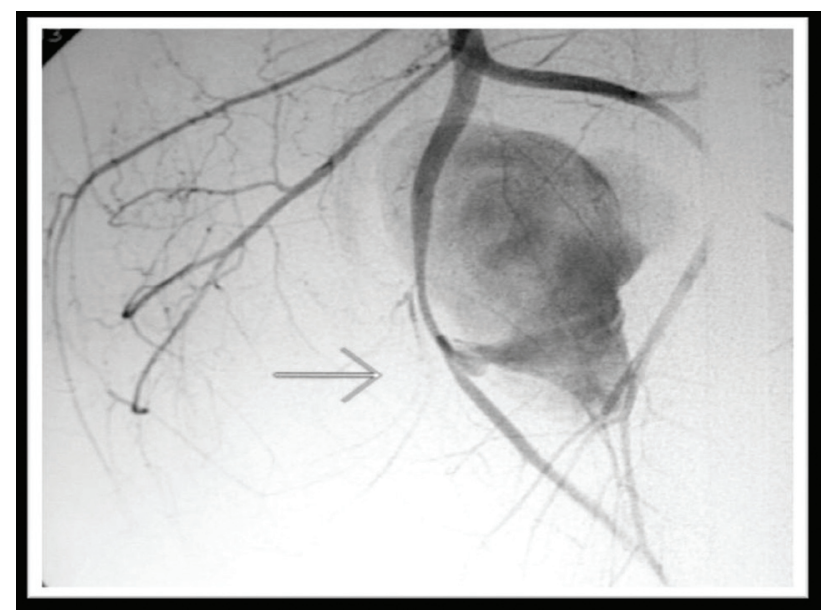

Figura 8. Arteriografia femoral profunda esquerda de paciente vítima de ferimento por arma branca em coxa, onde se observa volumoso pseudoaneurima secundário a lesão de um dos ramos nutrícios de coxa (seta)
Tabela 5. Critérios de seleção sugeridos para indicação do tratamento não-operatório no trauma hepático

\section{Critérios de seleção}

Estabilidade hemodinâmica

Exame físico abdominal normal

Integridade neurológica

Ausência de lesões intra-abdominais concomitantes

Mínima necessidade de hemotransfusão (até 2UI de concentrado de hemácias)

Estabilização da lesão nas tomografias abdominais subsequentes

Lesões hepáticas grau I e II podem ser conduzidas de forma conservadora e o trauma monitorado por meio de exames laboratoriais e de imagem seriados. Lesões grau III e IV podem ser tratadas por meio da embolização percutânea. Lesões de grau V e VI têm indicação formal para o tratamento cirúrgico (Tabela 6).

\section{Tabela 6. Classificação do trauma hepático segundo a Associação Americana de Cirurgia do Trauma}

\begin{tabular}{cll}
\hline Classe & Lesão & Descrição da lesão \\
\hline I & Hematoma & Subcapsular, não expansivo e extensão $<10 \%$ da superfície \\
II & Laceração & Ruptura capsular, sem sangramento e extensão $<1 \mathrm{~cm}$ \\
III & Hematoma & Subcapsular $>50 \%$ \\
III & Laceração & Profundidade $>3$ cm \\
IV & Hematoma & Sangramento intra-parenquimatoso \\
IV & Laceração & Desprendimento de $25-50 \%$ do parênquima \\
V & Laceração & Destruição $>50 \%$ do parênquima \\
V & Vascular & Lesões de veias hepáticas ou veia cava inferior retro-hepática \\
VI & Vascular & Avulsão hepática
\end{tabular}

Para a maioria das lesões hepáticas graves, o tratamento clássico foi o cirúrgico, mas, em 1983, Karp descreveu casos de crianças tratadas por meio da embolização percutânea com sucesso, o que foi corroborado em séries posteriores. Dado incidências entre 20 e 50\% de laparotomias não terapêuticas, avanços no diagnóstico por imagem e disseminação das técnicas endovasculares percutâneas, sobretudo a embolização, sugeriram a possibilidade do tratamento não operatório e a necessidade de es s cirúrgicos demonstrando tabelecer critérios de seleção para este tipo de trauma ${ }^{11}$ (Tabela 5).

As ressecções hepáticas são muitas vezes necessárias por conta da dificuldade em identificar sangramentos periféricos. Nestes casos, a embolização pode permitir a estabilização hemodinâmica do paciente ou evitar a cirurgia. O cateterismo superseletivo é importante para a identificação de colaterais que possam vir a perpetuar sangramentos. No caso de pseudoaneurismas, a oclusão seletiva não é recomendada, em função de paredes frágeis e maior risco de ruptura ${ }^{12}$ (Figura 9). 


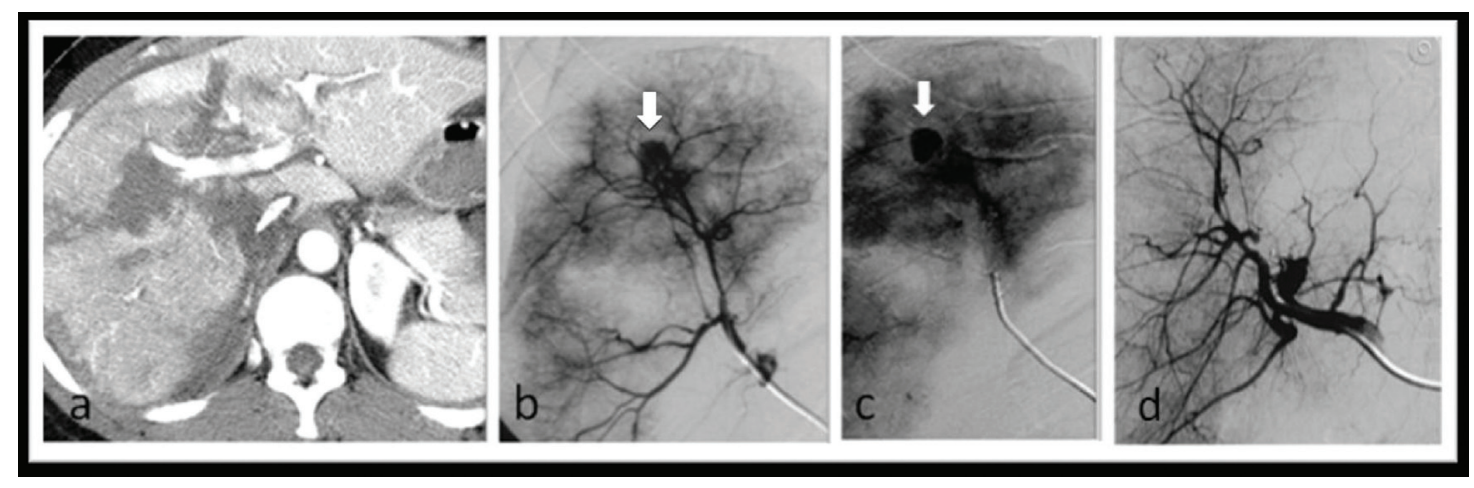

Figura 9. (a) Corte axial tomográfico de abdome superior em paciente vítima de trauma hepático contuso onde observase grande heterogeneidade, áreas de fraturas do parênquima e hematoma; (b, c e d) arteriografia hepática seletiva demonstrando na fase arterial, hetereogeneidade (áreas de contusão) e pseudoaneurisma (seta), que foi embolizado por meio do uso de molas metálicas com sucesso.

A oclusão da artéria hepática extra-hepática pode ser realizada em casos de ruptura, sempre que a veia porta se encontrar pérvia.

A avaliação pós-procedimento é realizada por meio de TC ou RM periódicas, indicandose o estudo angiográfico apenas na suspeita de ressangramentos.

\section{F. Lesões esplênicas}

O tratamento clássico das lesões esplênicas foi a esplenectomia até a década de sessenta.
Posteriormente, o tratamento conservador passou a ser adotado, principalmente depois da descrição de casos tratados com sucesso por meio de medidas não operatórias em crianças e adultos ${ }^{13}$.

As lesões esplênicas podem ser classificadas conforme o grau de lesão parenquimatosa ou vascular, a partir de onde se pode planejar adequadamente a conduta terapêutica (Tabela 7). Os critérios de seleção para o tratamento não operatório são semelhantes aos do trauma hepático.

\section{Tabela 7. Classificação das lesões esplênicas segundo a Associação Americana de Cirurgia do Trauma}

\begin{tabular}{ccl}
\hline Classe & Lesão & Descrição da Lesão \\
\hline I & Hematoma & Subcapsular, não expansivo e extensão $<10 \%$ da superfície \\
I & Laceração & Ruptura capsular, sem sangramento e profundidade $<1 \mathrm{~cm}$ \\
II & Hematoma & Subcapsular, não expansivo de 10 a $50 \%$ de superfície ou intraparenquimatoso $<2 \mathrm{~cm}$ \\
II & Laceração & Sangramento ativo e com 1 a $3 \mathrm{~cm}$ de profundidade \\
III & Hematoma & Expansivo ou subcapsular $>50 \%$ da superfície ou intraparenquimatoso maior que 2 cm \\
III & Laceração & Maior que 3 cm ou envolvendo vasos trabeculares \\
IV & Hematoma & Intraparenquimatoso roto ou com sangramento ativo \\
IV & Laceração & Vaso lobar ou destruição $>25 \%$ do parênquima \\
V & Vascular & Avulsão esplênica \\
\hline
\end{tabular}

A embolização arterial para tratamento da lesão esplênica é factível em, aproximadamente, 15 a $20 \%$ dos pacientes e a lesão mais frequente é o pseudoaneurisma. O controle pode ser realizado por meio da embolização seletiva ou pela oclusão troncular arterial esplênica com objetivo de diminuir a pressão de perfusão. Esta conduta não implica em isquemia do baço, porém, impossibilita re-embolizações ${ }^{14}$.

Este método é seguro, de baixo custo, baixa morbimortalidade e apresenta boas taxas de sucesso $(90 \%)$ em pacientes criteriosamente selecionados (Figura 10). 

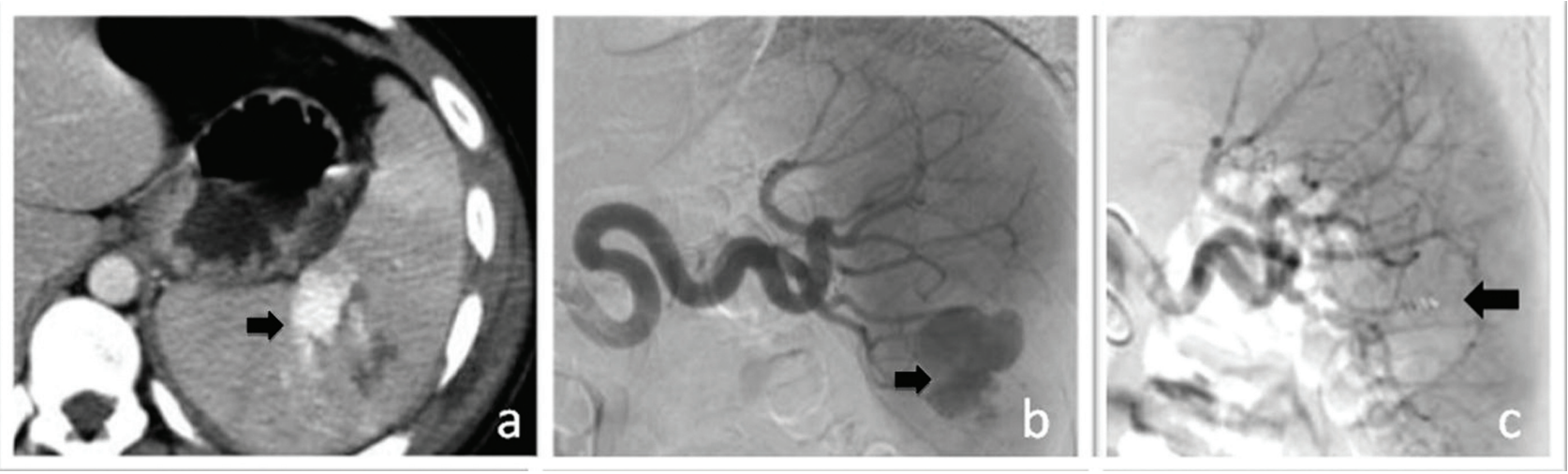

Figura 10. (a) Corte axial tomográfico de abdome superior em paciente vítima de trauma contuso onde se observa imagem de contraste extravascular, área de fratura do parênquima e hematoma (setas); (b e c) arteriografia esplênica seletiva demonstra, na fase arterial, extravasamento de contraste (foco de sangramento-seta), embolizado por meio do uso de molas metálicas (seta) com sucesso.

\section{G. Trauma renal}

O rim é lesado em cerca de $10 \%$ dos casos de traumatismo abdominal. A maioria (80\%) relaciona-se a traumas fechados, sendo que cerca de $90 \%$ destas lesões são classificadas como menores, podendo ser controladas sem cirurgia. Apenas 10 a $20 \%$ classificam-se como maiores, acometendo medula, pedículo vascular ou sistema coletor que resulta em extravasamento de urina ou maiores perdas volêmicas.

A adequada avaliação radiológica no trauma renal permitirá preservação do rim e de sua função, com mínimas sequelas.

O quadro clínico é variável, todavia, sinais como fratura dos últimos arcos costais, hematoma em flanco, contratura da musculatura abdominal e hematúria são sugestivos de lesão renal. Hematúria está presente em cerca de $90 \%$ dos casos e, embora não haja correlação com a intensidade e gravidade da lesão, ela é fator importante para indicação da investigação diagnóstica. A classificação do tipo de lesão renal em 5 graus é importante para a seleção de pacientes ${ }^{4}$ (Tabela 8 ).

A investigação diagnostica inclui urografia excretora, US, TC, RM e arteriografia. A arteriografia, além do potencial diagnóstico, permite realizar embolizações de vasos sangrantes, tratar fístulas arteriovenosas e preservar o rim. Está também indicada para casos de exclusão renal observada na urografia excretora, TC ou RM, quando há suspeita de trombose arterial.

O tratamento não operatório pode ser utilizado em $95 \%$ dos traumas fechados, em $50 \%$ dos penetrantes por arma branca e em cerca de 15\% das lesões por projétil de arma de fogo. A escoIha do tipo de tratamento, cirurgia ou embolização endovascular percutânea, deve ser realizada após criteriosa avaliação e discussão multidisciplinar que inclua radiologistas, cirurgiões do trauma, urologistas e radiologistas intervencionistas (Figura 11).

Tabela 8. Classificação das lesões renais segundo a Associação Americana de Cirurgia do Trauma

\begin{tabular}{cll}
\hline Classe & Lesão & Descrição da lesão \\
\hline I & $\begin{array}{l}\text { Hematoma/ } \\
\text { Laceração } \\
\text { II }\end{array}$ & Laceração \\
II & Hematoma & Cortical \\
III & Laceração & $\begin{array}{l}\text { Perinefrético não } \\
\text { expansível } \\
\text { Parenquimatosa } \\
\text { profunda com extensão } \\
\text { até a medula } \\
\text { Vasos renais principais }\end{array}$ \\
IV & Vascular & $\begin{array}{l}\text { Resulta em } \\
\text { desvascularização, } \\
\text { segmentar ou violam } \\
\text { sistema coletor }\end{array}$ \\
IV & Laceração & $\begin{array}{l}\text { Avulsão renal ou } \\
\text { trombose de artéria renal }\end{array}$ \\
& &
\end{tabular}

\section{H. Trauma pélvico}

As lesões pélvicas e de extremidades inferiores resultam nas mais altas taxas de incapacidade. Traumas de alta energia, como automobilísticos e quedas de alturas, podem produzir fraturas pélvicas de caráter instável com deslocamento do anel pélvico e hemorragia maciça ${ }^{15}$. Nesta condição, se medidas de controle forem retardadas, a grande perda volêmica determinará graves consequências.

As forças laterais podem deformar o anel pélvico e produzir fraturas acetabulares, mas estas 
são menos prováveis de determinar deslocamento com hemorragia maciça. Por sua vez, a rotação externa ou translação vertical ("vertical shear") das hemipelves pode determinar grande dano visceral, de tecidos moles e lesões vasculares com sangramento, bem como lesões ligamentares, instabilidade pélvica, lesões ureterais, vesicais e comprometimento do plexo lombo-sacro ${ }^{16}$.

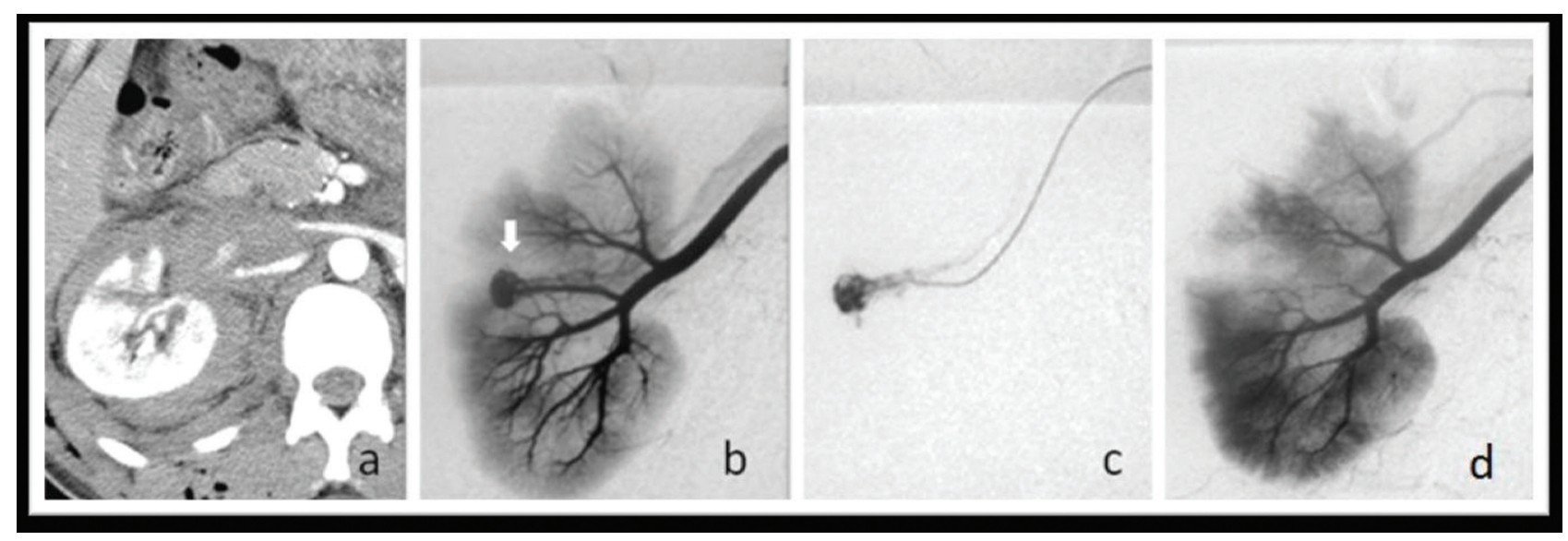

Figura 11. (a) Corte axial tomográfico em paciente vítima de trauma renal contuso à direita, onde se observa, heterogeneidade do parênquima e volumoso hematoma perirrenal direito. Em (b e c) arteriografia renal direita seletiva demonstra pseudoaneurisma em terço médio do rim (seta) e sua embolização como micromolas metáticas (c e d) com sucesso

O sangramento pélvico tem origem venosa em 85 a $90 \%$ e arterial em 10 a $15 \%$ dos casos. Apesar de o sangramento venoso ser de baixa pressão, a abertura do anel pélvico pode produzir grande sequestro de sangue para o retroperitônio, pré-vesical e intra-pélvico. Grandes quantidades de sangue também podem se acumular ao nível de bolsa escrotal, espaço pré-vesical e compartimentos musculares glúteos, sobretudo quando há ruptura e deslocamento da articulação sacro-ilíaca (rupturas posteriores do anel pélvico).

A rotação externa, por sua vez, lacera simultaneamente esses espaços, com acumulo de grande quantidade de sangue ${ }^{17}$.

O reconhecimento imediato da lesão e a redução mecânica para estabilização da pelve (manual, fixadores externos, internos ou pinça de Ganz) são medidas imprescindíveis para conter o sangramento venoso. O diagnóstico baseia-se na combinação do exame físico e estudo radiográfico. A persistência da instabilidade hemodinâmica, que sugere sangramento arterial, deve ser investigada por meio de angiografia seletiva e embolizada, se possível. As lesões vasculares arteriais costumam estar relacionadas aos pequenos ramos colaterais ilíacos internos, como artéria glútea superior, pudenda interna e sacral lateral, que são mais acometidas.

A indicação para embolização baseia-se na condição hemodinâmica e do grau de sangramento, devendo ser realizada quando o extravasamento de contraste é detectado. O uso de embolizantes temporários, como Gelfoam em pedaços com 2 a 4 $\mathrm{mm}^{3}$ de diâmetro, permite manter colaterais e cessar o sangramento. Os materiais de menor diâmetro envolvem um maior risco de isquemia. As molas podem ser utilizadas sozinhas ou em combinação ${ }^{18}$ (Figura 12).

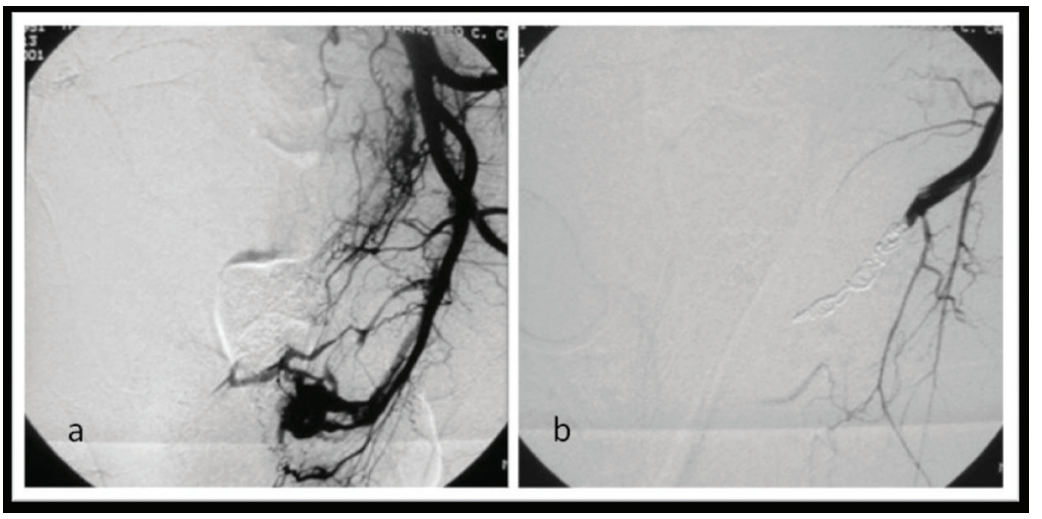

Figura 12. Em (a) arteriografia iliaca comum esquerda seletiva demonstrando volumoso pseudoaneurisma (seta) em paciente vitma de acidente automobilístico associado a trauma pélvico; (b) oclusão do tronco anterior da artéria iliaca interna após embolização com gelfoam e micromolas metálicas 
Normalmente, não é possível diminuir o fluxo venoso pélvico por meio da embolização arterial. $\mathrm{Na}$ ausência da identificação precisa do local do sangramento, embolizações não são recomendadas e deve-se considerar outras possíveis fontes de sangramento.

As complicações associadas à técnica incluem isquemia, infarto, infecção e disfunção erétil em pacientes do sexo masculino. Todavia, são pouco frequentes quando realizadas de forma seletiva. A embolização bilateral das artérias ilíacas internas é bem tolerada pela maioria dos pacientes.

\section{CONCLUSÕES}

- As técnicas radiológicas intervencionistas para o tratamento de focos hemorrágicos periféricos

\section{REFERÊNCIAS}

1. Trunkey DD. Trauma. Sci Am 1983;249:28-35.

2. Trunkey D. Torso trauma. Curr Probl Surg 1987; 24(4):209-65.

3. Jackson JE, Mitchell A. Advanced vascular interventional techniques in the management of trauma. Semin Int Rad. 1997;14(2):139-50.

4. Peitzman AB, Ferrada P, Puyana JC. Nonoperative management of blunt abdominal trauma: have we gone too far? Surg infect. 2009;10(5):427-33.

5. Parmley LF, Mattingly TW, Manion WC, et al. Nonpenetrating traumatic injury of the aorta. Circulation. 1958; 17:1087-101.

6. Frykberg ER. Advances in the diagnosis and treatment of extremity vascular trauma. Surg Clin North Am. 1995;75:207-23.

7. Weaver FA, Yellin AE. Complications of missed arterial injuries. J Vasc Surg. 1993;18:1077-8.

8. Vaughn GD, Mattox KL, Feliciano DV, et al. Surgical experience with expanded polytetrafluorethylene (PTFE) as a replacement graft for traumatized vessels. J Trauma. 1979;19:403-8.

9. Lovric Z, Wertheimer B, Candrlic K, et al. War injuries of major extremity vessels. J Trauma. 1994;36:248-51.

10. Weaver FA, Rosenthal RE, Waterhouse G, et al. ou viscerais são alternativas minimamente invasivas com ótimos resultados e alternativas às cirurgias de maior porte em pacientes vítimas de trauma;

- A radiologia intervencionista tem papel bem estabelecido para pacientes hemodinamicamente estáveis com lesão vascular periférica ou de órgãos sólidos;

- Estudos recentes sugerem que alguns pacientes instáveis podem se beneficiar dos tratamentos realizados pelos radiologistas intervencionistas, com consequente redução da necessidade de cirurgia;

- Evidências científicas comparando o uso das técnicas radiológicas intervencionistas com a cirurgia, em casos de trauma, são ainda escassas.

Combined vascular and skeletal injuries of the lower extremities. Am Surg. 1984;50:189-97.

11. Hagiwara A. Nonsurgical management of patients with blunt hepatic injury: efficacy of transcatheter embolization. AJR. 1997;169;1151-6.

12. Karp MP. Cooney DR, Pros GA, et al. The nonoperative management of pediatric hepatic trauma. J Pediatr Surg, 1983;18:512-8.

13. Pahter HL, Spencer FC, Hofstetter SR, et al. Experience with selective operative and nonoperative treatment of esplenic injuries in 193 patients. Ann Surg. 1990;211:583-91.

14. Shackford SR, Molin M. Management of splenic injuries. Surg Clin North Am. 1990;70:595-620.

15. Huittinem VM, Slatis P. Postmortem angiography and dissection of the hypogastric artery in pelvic fractures. Surgery. 1973;73:454-62.

16. Mucha $P$, Farnell MB. Analysis of pelvic fracture management. J Trauma. 1984;24:379-86.

17. Kam J, Jackson H, Ben-Menchem Y. Vascular injury in blunt pelvic trauma. Radiol Clin North Am. 1981;19:17186.

18. Eger M, Goldman L, Goldstein A, et al. The use of a temporary shunt in the management of arterial vascular injuries. Surg Gynecol Obstet. 1971;123:67-70. 